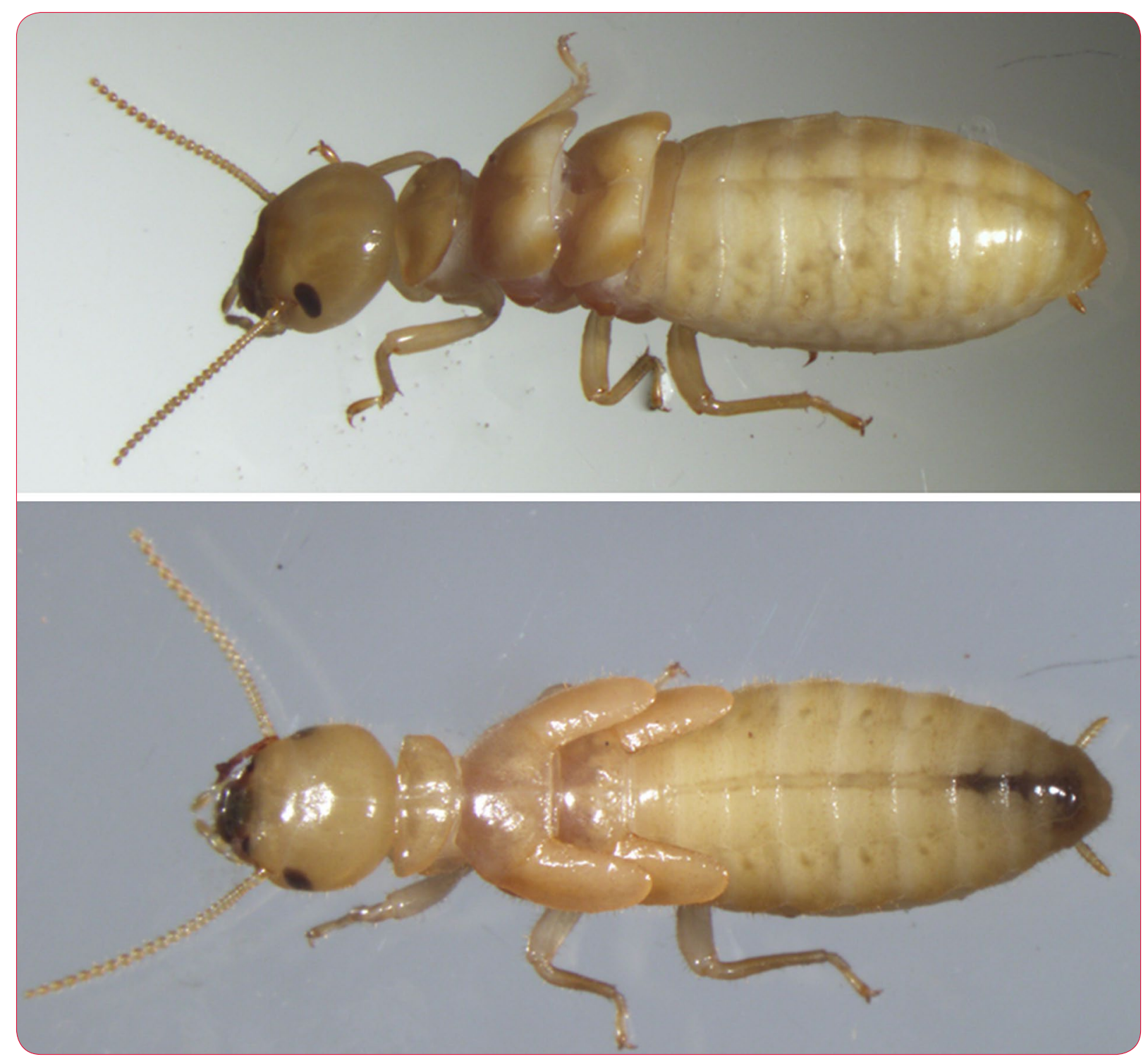

Reduction of a nymphal instar in a dampwood termite: heterochronic shift in the caste differentiation pathways

Nii et al. 


\title{
Reduction of a nymphal instar in a dampwood termite: heterochronic shift in the caste differentiation pathways
}

\author{
Ryotaro Nii ${ }^{1}$, Kohei Oguchi ${ }^{2}$, Junpei Shinji ${ }^{2}$, Shigeyuki Koshikawa ${ }^{3}$ and Toru Miura ${ }^{2 *}$
}

\begin{abstract}
Background: Generally in termites, alates differentiate through multiple nymphal instars which gradually develop wing buds. However, in a dampwood termite, Hodotermopsis sjostedti, alates molt directly from a single nymphal instar with short wing buds. In this study, to examine the mechanism underlying the wing formation during the alate differentiation in H. sjostedti, histological and morphological observations were carried out on the developmental process of wing formation during the nymphal instar, in comparison with those in Zootermopsis nevadensis, which has two nymphal instars. Furthermore, the expression patterns of genes that are thought to be responsible for wing formation, i.e., wing-patterning genes and genes encoding hormone-related factors, were quantified during alate differentiation and compared between the two species.

Results: The results showed that, in H. sjostedti, wings were formed in a complicatedly folded shape, not only inside the wing buds as seen in Z. nevadensis, but also under the dorsal thoracic cuticle, where the wing tips shifted toward the median thoracic part. Accordingly, the wing expansion pattern also differed from that in Z. nevadensis. Furthermore, the results of real-time qRT-PCR on overall expression profiles of wing-patterning genes and hormone-related genes suggest that the single nymphal instar in $\mathrm{H}$. sjostedti well resembles to the second nymphal instar in Z. nevadensis. In particular, significant upregulation of vestigial ( $\mathrm{vg}$ ) and downregulation of Krüppel homolog 1 (Kr-h1) that were observed at the second nymphal instar in Z. nevadensis apparently occurred during the single nymphal instar in $H$. sjostedti.
\end{abstract}

Conclusion: The developmental events for wing formation are compacted into a single nymphal instar in $\mathrm{H}$. sjostedti, and as a result, the unique wing formation is seen to compensate for the spatial restriction inside small wing buds, leading to the completion of functional wings.

Keywords: Termites, Alate differentiation, Caste differentiation, Nymphal instar, Wing formation, Gene expression, Heterochrony

\section{Background}

Eusocial insects such as bees, wasps, ants and termites show highly organized sociality with divisions of labor among morphologically and behaviorally differentiated castes [1]. Patterns of caste differentiation in termites differ from those in social hymenopterans, mostly due to

\footnotetext{
*Correspondence: miu@mmbs.s.u-tokyo.ac.jp

${ }^{2}$ Misaki Marine Biological Station, School of Science, The University of Tokyo, Miura, Kanagawa 238-0225, Japan

Full list of author information is available at the end of the article
}

the modes of postembryonic development: hemimetabolous in termites and holometabolous in hymenopterans [2]. In termites, the number of instars can vary among species and even larval (immatures without wing buds) and nymphal instars (immatures with wing buds) can perform colony tasks, so that soldiers and workers possess immature characteristics [3]. Therefore, the caste differentiation pathways are diverse among termite lineages, suggesting that the pattern of postembryonic development, i.e., molting and morphogenesis, has evolved in association with social evolution (Fig. 1). 


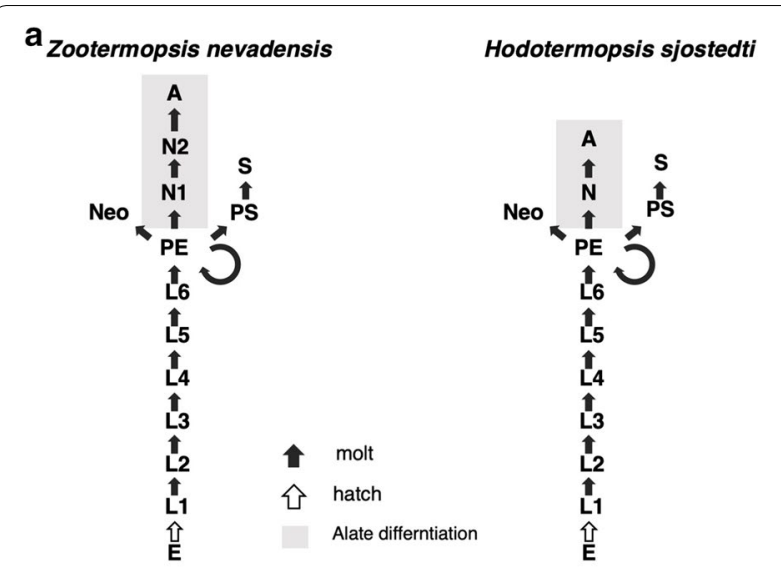

b Zootermopsis nevadensis

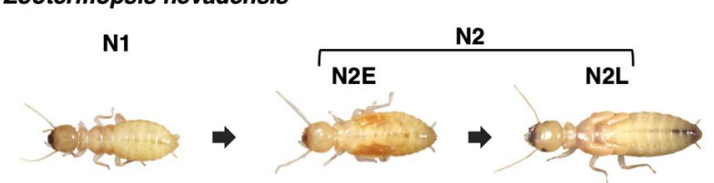

Hodotermopsis sjostedti

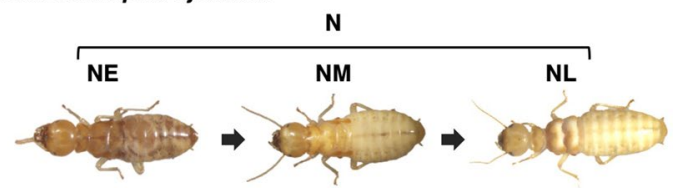

Fig. 1 a Caste differentiation pathways in the two termite species studied here: Zootermopsis nevadensis (left) and Hodotermopsis sjostedti (right). Abbreviations are: E, egg; L, larva, i.e., immatures without wing buds; PE, pseudergate; N, nymph, i.e., immatures with wing buds/pads; A, alate; Neo, neotenic; PS, presoldier; S, soldier. b Nymphal stages of the two species that were focused on in this study. In this study, the last nymphal instar was divided into several stages in order to understand the developmental processes. Abbreviations are: N1, first-instar nymph; N2E, early second-instar nymph; and N2L, late second-instar nymph in Z. nevadensis, and NE, early nymph; NM, mid-nymph, and NL, late nymph in H. sjostedti

The caste differentiation pathways can be classified into two major patterns depending on the timing of differentiation into reproductive individuals (imagos): linear and forked pathways [3-5]. In the forked pathways, alate development proceeds exclusively via nymphal instars, while worker and soldier development proceed via distinct larval instars. In contrast, in the linear pathways, all individuals go through larval instars without wing buds up to late instars (i.e., pseudergates that perform worker tasks but still possess potential to develop into alates). A fraction of these pseudergates differentiate into alates through nymphal instars with apparent wing buds or wing pads $[4,6,7]$. The forked pathways are seen in Mastotermitidae, Hodotermitidae, and most species of Rhinotermitidae and Termitidae, while the linear pathways are seen in Archotermopsidae, Kalotermitidae, Serritermitidae and a part of Rhinotermitidae [3, 8].

In termites, although the number of moltings that are required for the soldier differentiation is constant among species ( 2 moltings via presoldier), the number of nymphal instars is diverse among termite lineages $[4,6,7]$. Generally, termites possess multiple nymphal instars through which wing buds are gradually developed, forming complete wings [3]. For example, in Termitidae showing the forked pathways, there are 6 nymphal instars [9]. Species belonging to Archotermopsidae, which are thought to have primitive characteristics, possess 2 or more successive nymphal instars $[4,10]$.

However, in the Japanese dampwood termite Hodotermopsis sjostedti, which belongs to Archotermopsidae, is distributed in parts of East and Southeast Asia, pseudergates differentiate into alates through only a single nymphal instar with short wing buds (Fig. 1, [11, 12]). Other related species such as Zootermopsis nevadensis (Archotermopsidae) possess 2 nymphal instars, i.e., short and long wingbudded nymphs (Fig. 1, [10]). In these species, wing buds gradually develop and alate wings form inside the long wing buds (wing pads) at the final nymphal instar. This suggests that the developmental process of alate differentiation in $H$. sjostedti is distinctive, since wings should be formed inside the small wing buds of the first-instar nymphs.

In this study, therefore, to clarify the mechanism underlying the wing formation during the alate differentiation in $H$. sjostedti, histological and morphological observations were carried out on the developmental process of wing formation during its nymphal instar, in comparison with those in $Z$. nevadensis. Furthermore, expression patterns of genes that are thought to be responsible for the wing formation, i.e., wing-patterning genes and genes for hormone-related factors, were quantified during the course of alate development and compared between the two species. The regulatory genes involved in wing formation are known in several insects (e.g., [13-15]). In addition, hormone-related factors involved in molting and metamorphosis have also been reported in many insects (e.g., [16-20]). Altogether, 5 wingpatterning genes and 9 hormone-related genes were identified, and their expression patterns were analyzed during alate differentiation in the two focal species. Results clearly showed that the overall expression patterns at the second nymphal instar in $Z$. nevadensis apparently shifted to the single nymphal instar in $H$. sjostedti.

\section{Results}

\section{Histological observations during wing formation}

Based on histological observations on paraffin sections of thoracic parts of nymphs, the developmental patterns of wings inside wing buds were compared between $H$. sjostedti and $Z$. nevadensis (Fig. 2). In Z. nevadensis, short 


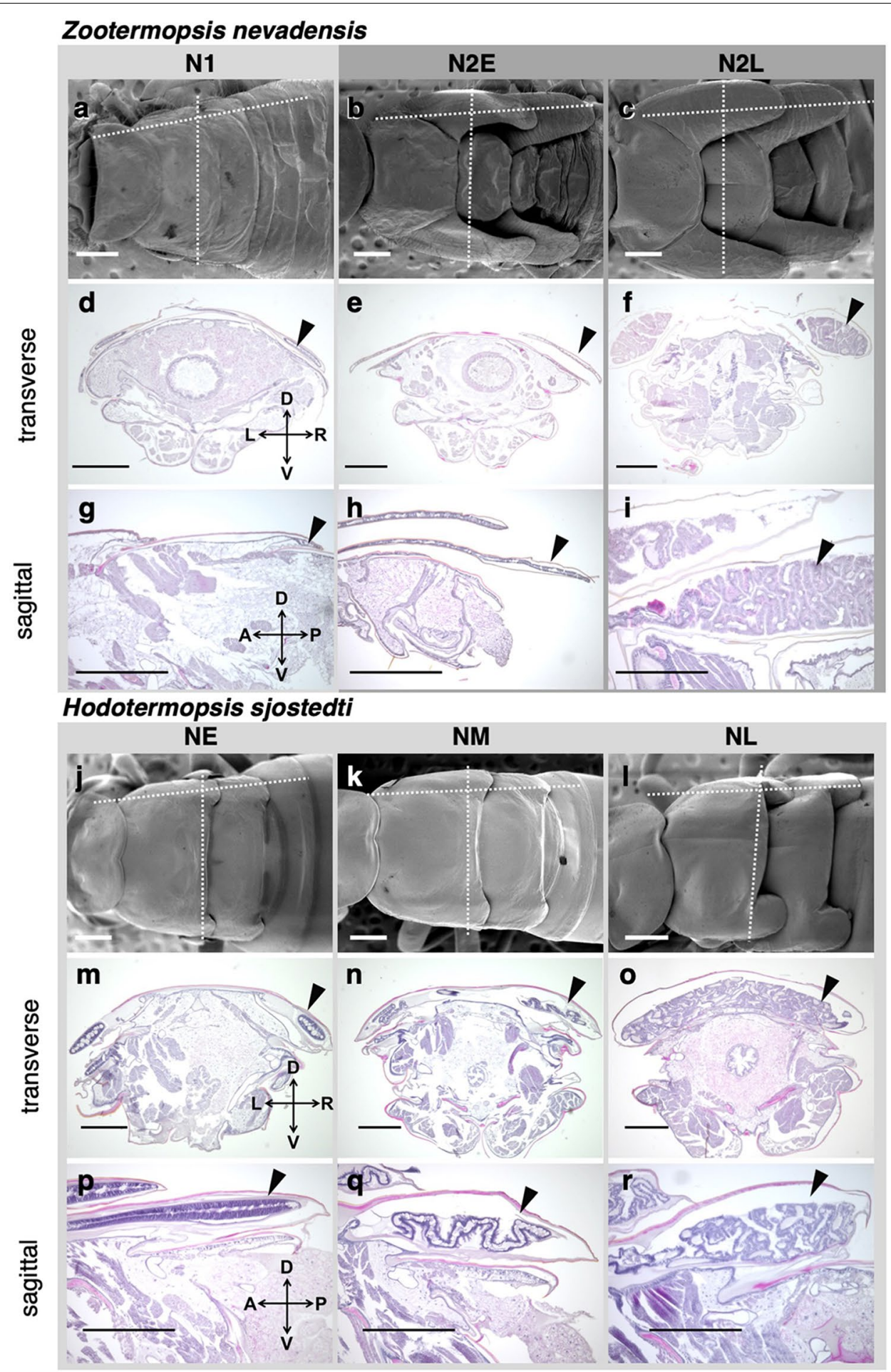

Fig. 2 Morphological and histological transitions during nymphal instars in Z. nevadensis (a-i) and H. sjostedti (j-r). Developmental processes of nymphal instars are divided into three stages: N1 (first-instar nymph; $\mathbf{a}, \mathbf{d}, \mathbf{g}$ ), N2E (early second-instar nymph; $\mathbf{b}, \mathbf{e}, \mathbf{h}$ ) and N2L (late second-instar nymph; $\mathbf{c}, \mathbf{f}, \mathbf{i}$ ) in Z. nevadensis; and NE (early nymph; $\mathbf{j}, \mathbf{m}, \mathbf{p}$ ), NM (mid-nymph; $\mathbf{k}, \mathbf{n}, \mathbf{q}$ ), and NL (late nymph; $\mathbf{I}, \mathbf{o}, \mathbf{r}$ ) in H. sjostedti. Arrowheads indicate epithelial tissues of developing wings. All scale bars indicate $500 \mu \mathrm{m}$ 
wing buds of the first-instar nymphs (N1) elongated to become long wing buds through a molt into the secondinstar nymphs (N2) (Fig. 2a-c). Inside the wing buds, a thin layer of epithelial tissue was seen (Fig. 2d, e, g, h). Prior to the molt into alates, epithelial tissues proliferated extensively and the layer was folded together with the newly formed cuticular layer inside the wing buds (Fig. 2f, i).

In the case of $H$. sjostedti, at the early stage of nymphs (NE), a single layer of epithelial tissue was observed under the cuticle of short wing buds (Fig. $2 \mathrm{~m}, \mathrm{p}$ ). Later, at the mid-stage of nymphs (NM), the epithelial layer started to proliferate, forming a folded structure (Fig. 2n, q). Then, at the late stage of nymphs (NL), the folded structure became thicker and more complicated (Fig. 2o, r). The proliferated epithelial tissues that would form wings could not be contained inside the wing buds. So, as a result, the tissues of folded epithelial layers were seen in the median region of thoracic parts (Fig. 2o).

\section{SEM observations on wing expansion}

SEM observations of the thoracic region at the time of imaginal molt showed clear differences in the process of expansion of alate wings between the two species (Fig. 3). In Z. nevadensis, the folded structures of wings are only seen inside wing buds, even after the completion of epithelial proliferation just prior to the imaginal molt (Fig. 3a). A major groove is seen in the middle of each folded wing. After the onset of imaginal molt, folded wings started to expand from the basal part (Fig. 3b). Then, at the latest stage of wing expansion, most of the wings were expanded, except that only an apical part of the wing was still folded (Fig. 3c). This process of wing expansion showed that the apical positions of wings were formed at the tips of wing buds.

On the other hand, in H. sjostedti, since folded wings could not be contained inside the short wing buds of nymphs, the folded wings extended into the median region of the thoracic part (Fig. 3d), as seen in the section images (Fig. 2o). After the onset of wing expansion, as seen in $Z$. nevadensis, wings were expanded from the basal part, so that the folded shape of the apical part of the wing was maintained (Fig. 3e, f). These results showed that, in the case of $H$. sjostedti, the apical part of the wing did not form at the tip of the wing bud, but rather at a position between the wing-bud tip and the median line of the thorax.

\section{Expression patterns of wing-patterning genes and hormone-related genes}

Using real-time qRT-PCR, the expression patterns of wing-patterning genes during nymphal stages were compared between the two species (Fig. 4). In Z. nevadensis,

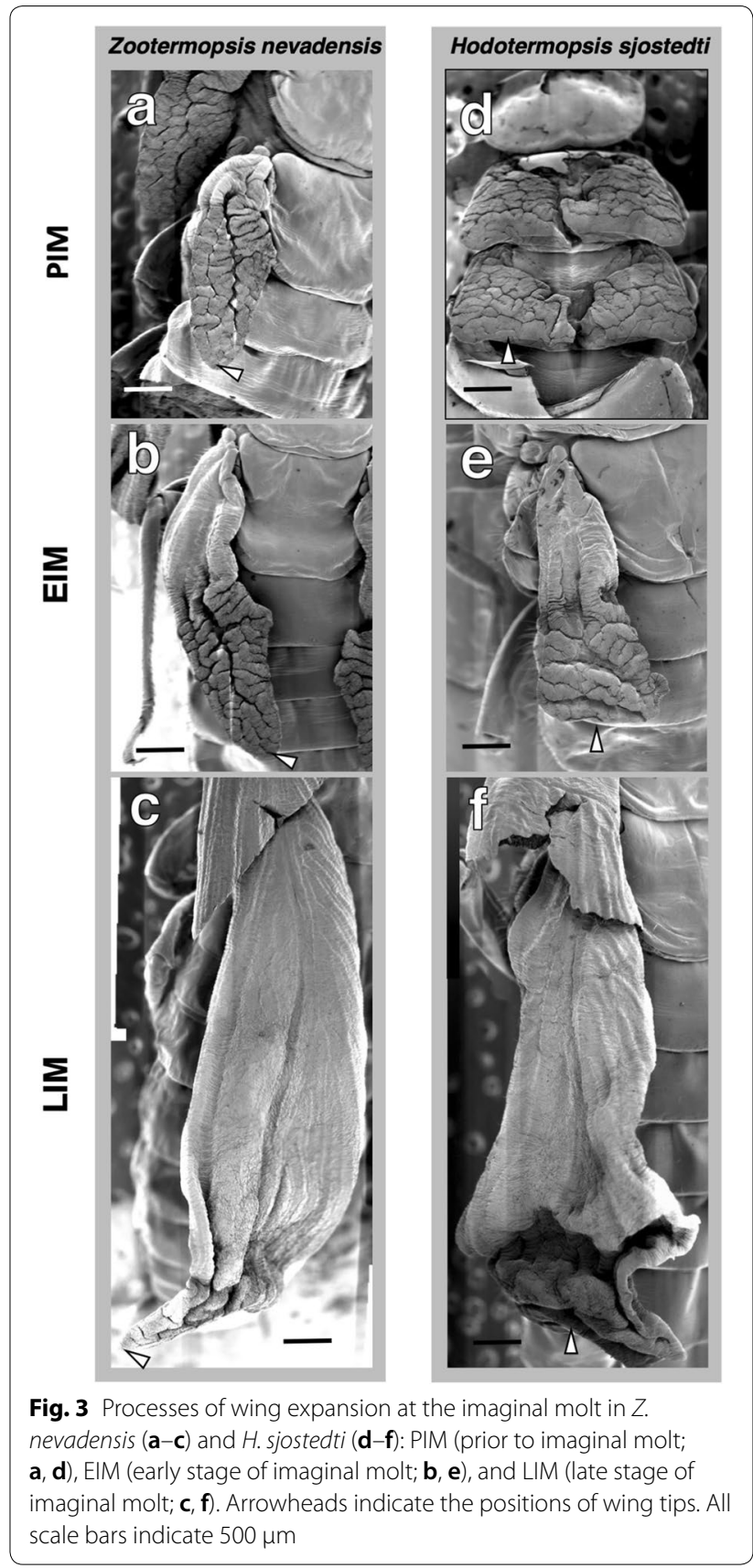

significant differences among developmental stages were only detected for the vestigial $(v g)$ gene (Fig. 4c), although most of the regulatory genes were slightly upregulated at the N2E stage. In H. sjostedti, vg, decapentaplegic (dpp), extradenticle (exd) and Sex-combs reduced (Scr) showed significant differences among developmental stages (Fig. 4b-e; $P<0.05$, Tukey's test after one-way ANOVA). $d p p$, exd and $S c r$ were upregulated at the NE stage, while $v g$ was upregulated at the NL stage. Overall, expression peaks were observed at N2E in Z. nevadensis and at NE 


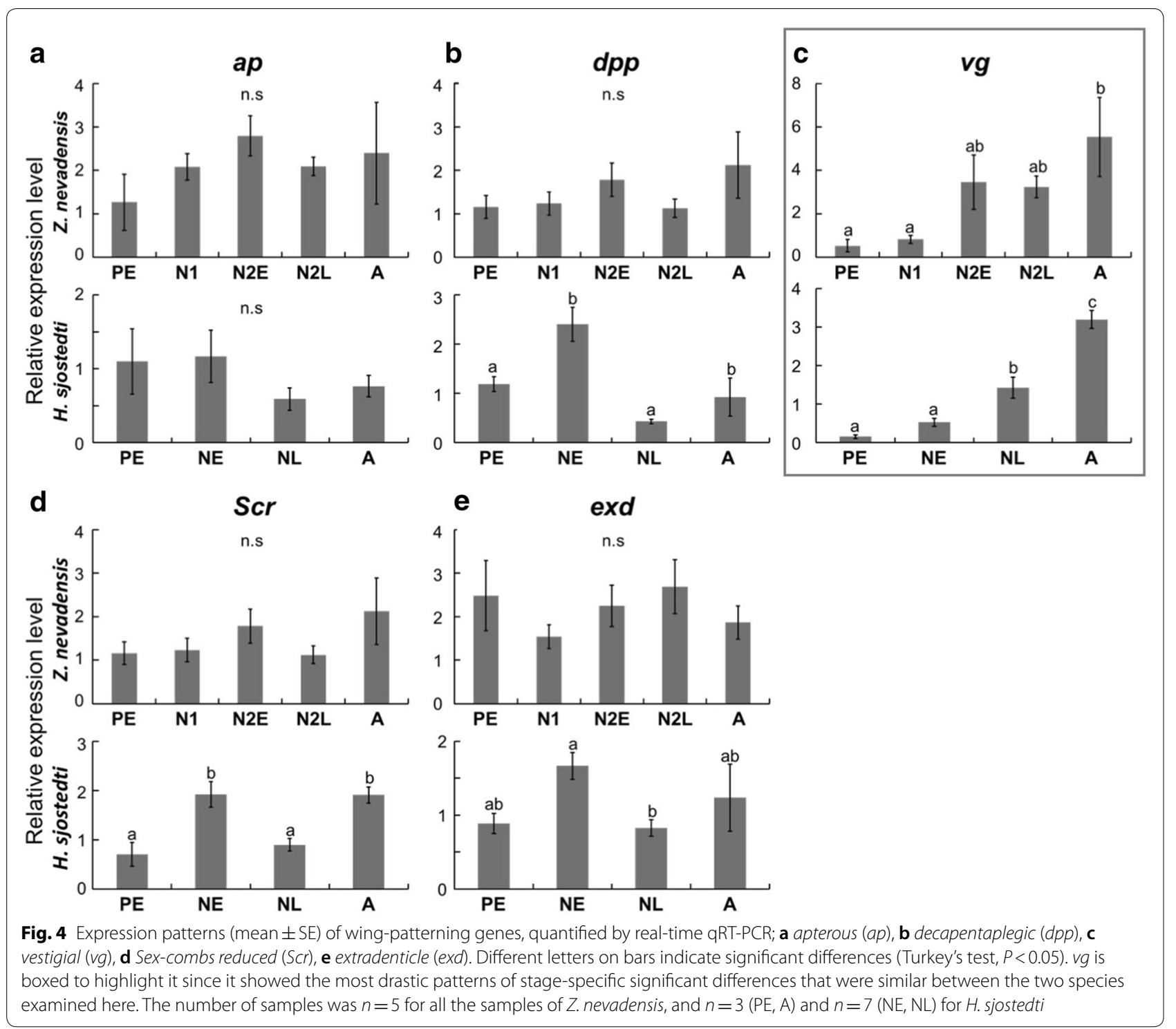

in $H$. sjostedti. In addition, the expression levels in alates (A) were also high in both species. Only $v g$ showed a different pattern from other genes, namely, upregulation just before the imaginal molt.

Hormone-related genes showed more complicated stage-related expression patterns (Fig. 5). Although clear significant differences were not detected, most of the genes examined (Methoprene-tolerant [Met], Broadcomplex [BR-C], Ecdysone Receptor [EcR], ultraspiracle [USP], Insulin Receptor 2 [InR2], Forkhead box $O$ $[F O X O])$ showed similar expression patterns to wingpatterning genes, namely expression peaks were seen in $\mathrm{N} 2 \mathrm{E}$ in Z. nevadensis, and in NE in H. sjostedti (Fig. 5a, $\mathrm{c}-\mathrm{e}, \mathrm{h}, \mathrm{i})$. On the other hand, Krüppel homolog 1 (Kr-h1) showed distinctive patterns in both species; it was clearly downregulated from N2L in Z. nevadensis, and from NL in $H$. sjostedti (Fig. 5b). EcR and USP showed significant downregulation at NL in $H$. sjostedti (Fig. 5d, e). E93 and E75 showed significant upregulation at N2L in $Z$. nevadensis, but no significant differences among stages in H. sjostedti (Fig. 5f, g). Insulin signaling factors, InR2 and FOXO, showed similar expression patterns in the two species: upregulation at $\mathrm{N} 2 \mathrm{E}$ or $\mathrm{NE}$ and downregulation at N2L or NL (Fig. 5h, i). Many of the genes (Met, BR$C, E c R, U S P, E 93, I n R 2, F O X O)$ showed relatively high expression levels at A (Fig. 5a, c-f, h, i).

Principal component analysis on gene expression patterns With all the genes examined in this study, principal component analysis was carried out to compare the overall 


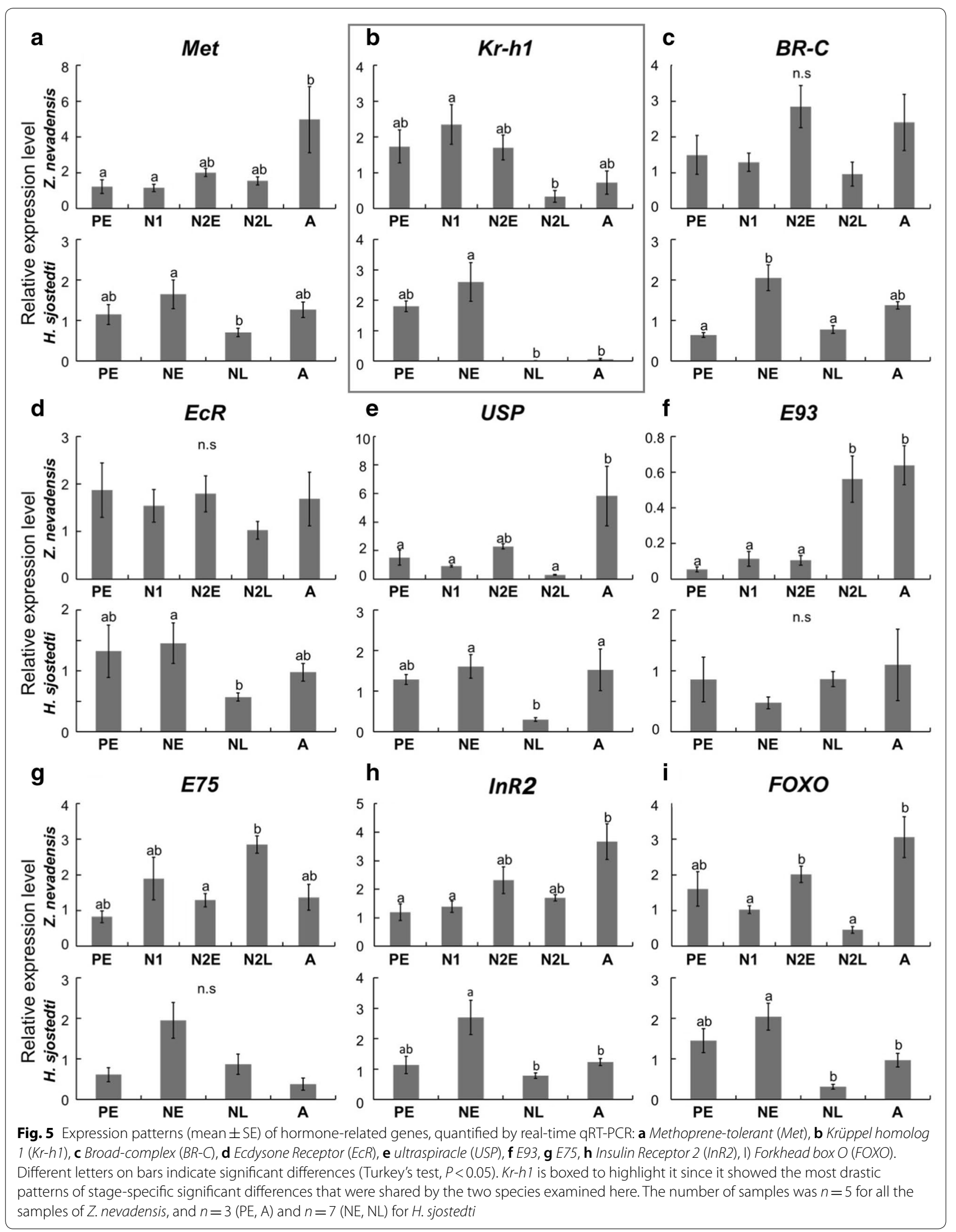


a

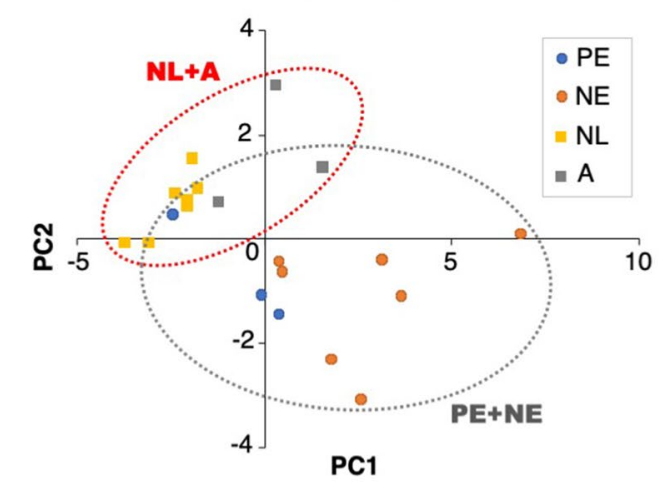

b 3] Hodotermopsis sjostedti

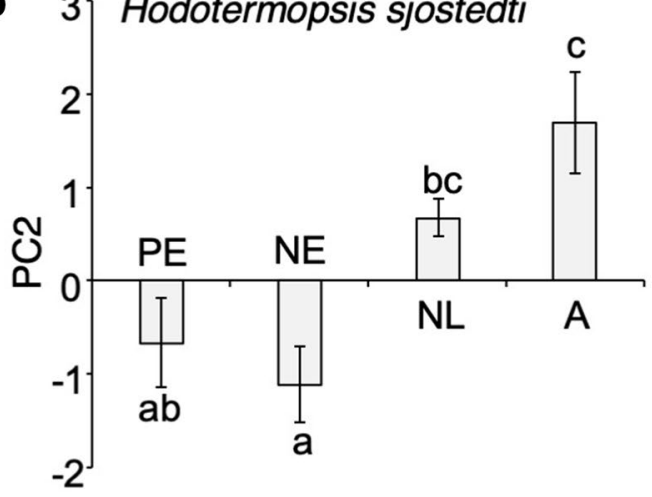

\section{Zootermopsis nevadensis}
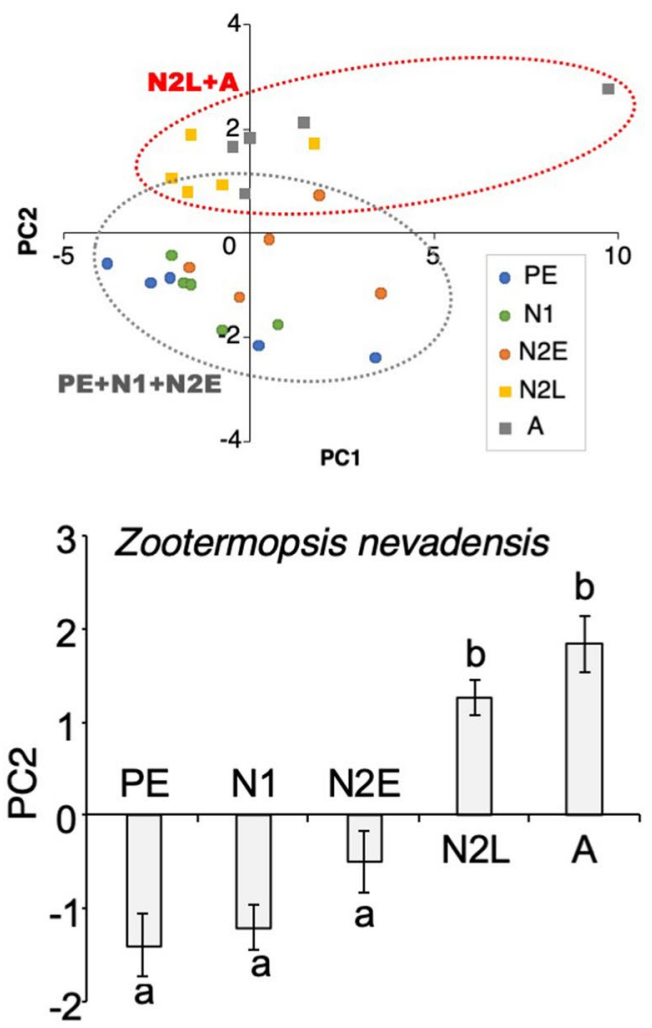

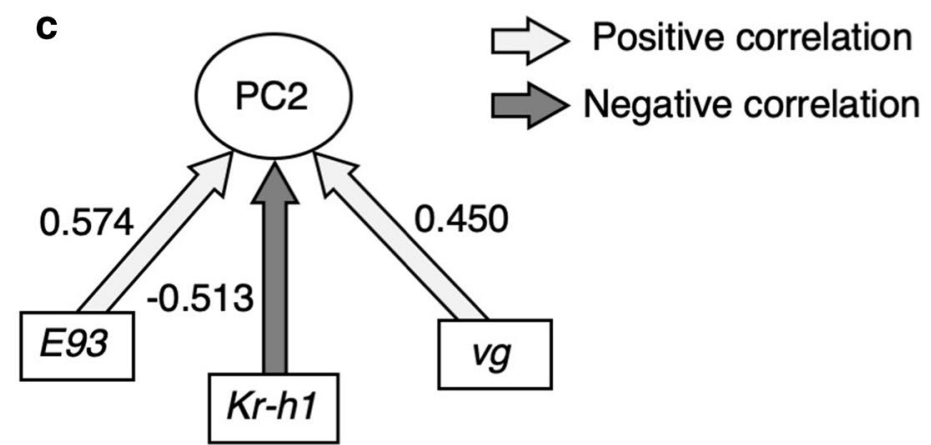

Fig. 6 Results of principal component analysis (PCA) based on the gene expression data. a Biplots of the principal component scores of examined stages during alate differentiation (PC1 vs. PC2), based on the results of the GLM analysis. The red dotted circles indicate the plots of NL and A in H. sjostedti or N2L and A in Z. nevadensis. The gray dotted circles indicate the other stages. $\mathbf{b}$ Principal component scores of PC2 in the respective stages in the two species. Bars indicate standard errors, and alphabetical letters indicate significant differences $(n=3-7$, Tukey-Kramer test, $P<0.05)$. c Schematic model explaining the impacts of expression levels of 3 genes showing higher PC loadings

gene expression patterns between the two species (Fig. 6). To predict which nymphal stage in $Z$. nevadensis corresponds to that in $H$. sjostedti, hypothetical nymphal stage models were made based on the stages in $H$. sjostedti, and they were statistically examined in $Z$. nevadensis. As the results, 4 principal components (PCs) were selected
(Additional file 1: Fig. S1), and the cumulative proportion of the 4 PCs was 0.814 , that sufficiently explains the variances of the data (Additional file 2: Table S1). Although GLM analysis showed that the PC1 and the PC3 depended on the species difference, the PC2 and the PC4 were shown to be independent from the species 


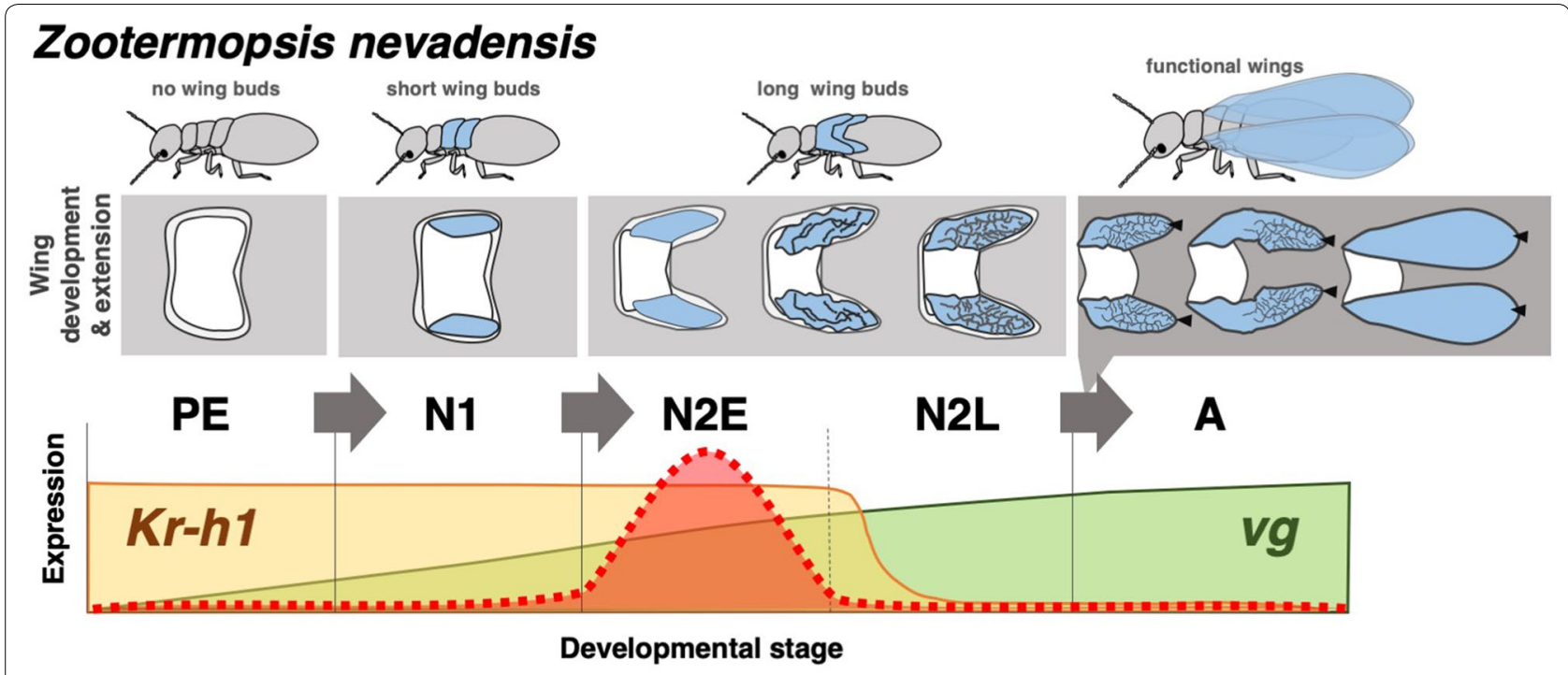

\section{Hodotermopsis sjostedti}

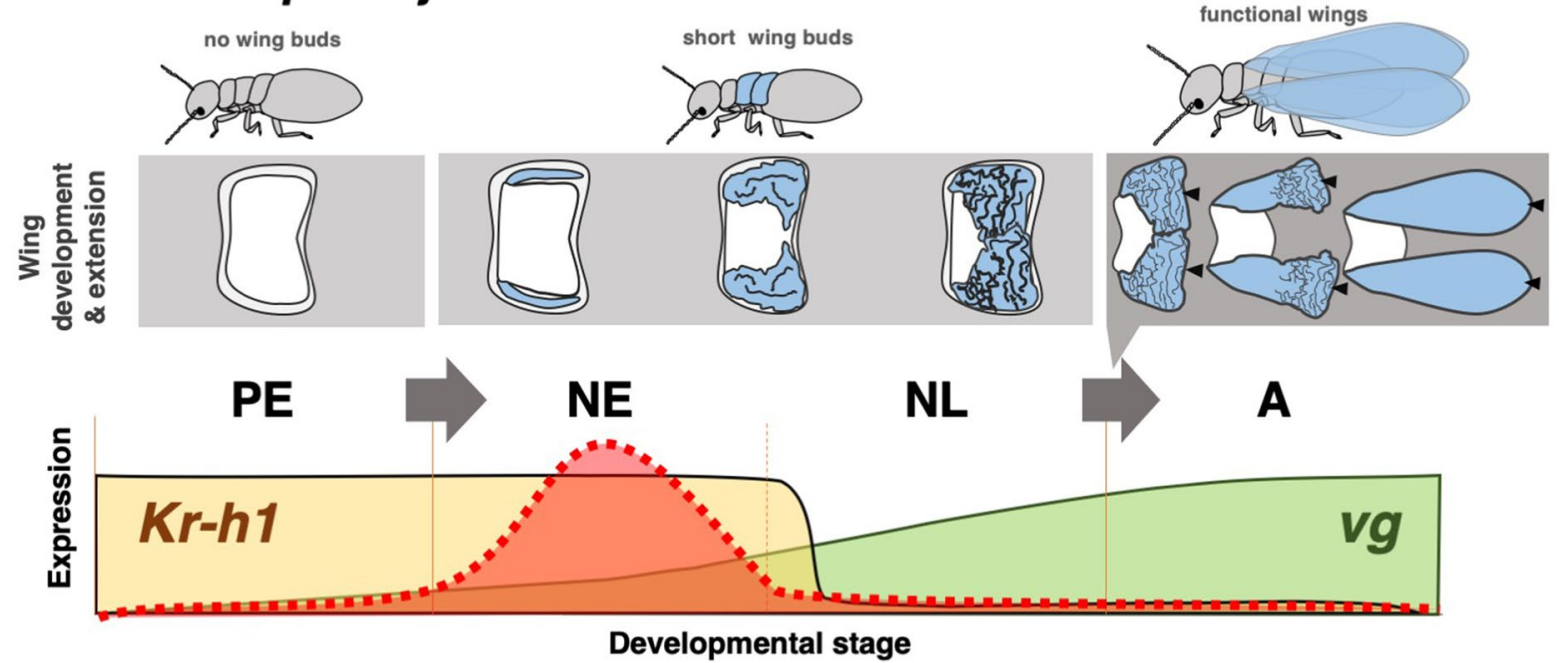

Fig. 7 Summary diagrams of wing development and gene expression patterns in Z. nevadensis and H. sjostedti determined in this study. The processes of wing formation are indicated along the course of nymphal development, followed by the wing expansion at the time of imaginal molt (top row for each species). Expression patterns of representative genes ( $\mathrm{g} g$ and $\mathrm{Kr}-\mathrm{h} 1$ ) are indicated: $\mathrm{Kr}-\mathrm{h} 1 \mathrm{is}$ abruptly downregulated at N2L and $\mathrm{NL}$, while $\mathrm{vg}$ is gradually upregulated at the late stage of the final-instar nymphs. Although statistically significant differences were not detected, expression peaks of most examined genes were seen at N2E in Z. nevadensis, while they were seen at NE in H. sjostedti (dotted lines)

difference (Additional file 3: Fig. S2, Additional file 4: Table S2; $n=45$; Wald test; $P<0.05)$. The PC2 scores increased at NL and A in $H$. sjostedti, the N2L and A in Z. nevadensis (Fig. 6a, b; $n=3-7$; Brown-Forsythe test; $P>0.05$; one-way ANOVA; $P<0.05$; Tukey-Kramer test; $P<0.05$ ) (Additional file 5: Table S3; Additional file 4: Table S2). In addition, the expressions of E93, $K r-h 1$ and $v g$ showed higher contribution ratio to PC2 (Fig. 6c, Additional file 2: Table S1). These indicate that the PC2 well explains the gene expression patterns during the nymphal stages.

\section{Discussion}

The differences of wing formation

As shown by the results obtained in this study, in $H$. sjostedti, the imaginal molt to alates occurs from the first-instar nymphs with short wing buds. Therefore, the developmental sequence of events of its wing formation differs from that of related species that possess multiple nymphal instars, such as $Z$. nevadensis. Histological and morphological investigations revealed that, in $H$. sjostedti, the extensive epithelial cell proliferation starts during the short-winged nymphal instars so that the complicatedly 
folded epithelial tissues are compacted all over the thorax (Fig. 2). As a result, the folding and expansion patterns of wings differ from those in Z. nevadensis (Fig. 3).

\section{Heterochronic shifts in wing-patterning and hormone-related genes}

From these patterns of epithelial proliferation and wing expansion, it was hypothesized that the expression of genes for wing formation should occur earlier (in shortwing-budded first-instar nymphs) in $H$. sjostedti, as the result of deletion of a nymphal stage. As predicted, this study revealed that many of the genes required for wing formation are upregulated in the second nymphal instar of $Z$. nevadensis, but in the first nymphal instar of $H$. sjostedti (Figs. 4, 5). Interestingly, the pattern of $v g$ expression was different from other genes (later in development), probably due to the functional differences among genes. The RNA extraction was carried out from whole thoracic segments, so that these expressions can be responsible not only for the wing development, but also for other tissues including flight muscles, which are also required for alate development. In conclusion, it can be said that heterochronic shifts of wing formation have occurred in the lineage of $H$. sjostedti, so that the developmental events for wing formation that normally happen through two (or more) nymphal instar stages have been compacted within a single nymphal instar.

In endocrine systems in insects, in general, downregulation of the juvenile hormone $(\mathrm{JH})$ pathway is required for the imaginal molt [21]. Therefore, also in termites, a low $\mathrm{JH}$ level is known to induce alate differentiation [2224]. We showed in the present study that the expression level of $K r-h 1$ was maintained until the early stage of second nymphal instar (N2E) and downregulated at the late stage (N2L) in $Z$. nevadensis, while it was downregulated at the late stage of nymphs (NL) in $H$. sjostedti, suggesting that the imaginal molt is apparently induced during the short-wing-budded nymphal instar in $H$. sjostedti. This situation is similar to the precocious metamorphosis reported in some hemimetabolous insects, in which knockdown of $K r-h 1$ induced precocious metamorphosis $[17,25,26]$. It is therefore suggested that, during evolution, some adventitious downregulation of $\mathrm{JH}$ signaling may have led to a precocious imaginal molt, leading to the lack of second-instar nymphs in $H$. sjostedti.

Among genes that showed no significance in the expression levels, many genes such as $d p p, S c r, B R-C$, USP, E75, InR2 and FOXO, showed interesting patterns that can explain the heterochronic shifts; NE and NL in $H$. sjostedti, respectively, corresponds to $\mathrm{N} 2 \mathrm{E}$ and $\mathrm{N} 2 \mathrm{~L}$ in $Z$. nevadensis. As for the overall expression patterns, the results of PCA clearly supported the ideas of heterochrony (Fig. 6). All the principal components independent from the species differences supported that $\mathrm{N} 1$ and $\mathrm{N} 2 \mathrm{E}$ in $Z$. nevadensis corresponds to NE in $H$. sjostedti, and N2L in Z. nevadensis corresponds to NL in H. sjostedti (Additional file 4: Table S2).

\section{The developmental constraints of short wing buds}

Also in other species, i.e., Prorhinotermes inopinatus and Termitogeton planus, a single nymphal instar was reported, although the nymphs possess relatively long wing buds $[27,28]$. It is also reported that, in Nasutitermes princeps, the number of nymphal instars can change depending on extrinsic factors, inducing short-winged alates called "microimagos" that differentiate from a younger nymphal instar [29]. Although their wings are non-functional, they reproduce as replacement reproductives. However, in the case of $H$. sjostedti, although it has only a single nymphal instar with short wing buds, the alate wings are fully functional. Therefore, this species is suggested to have overcome the developmental constraints caused by the lack of second-instar nymphs (Fig. 7).

\section{Adaptive significance and intra-colonial conflict}

Considering the adaptive significance underlying this unique developmental phenomenon, a candidate factor is thought to be intra-colonial conflict among reproductive candidates. As previously suggested [30], wing bud mutilation could be related to the intra-colonial conflict over reproduction. Mutilated nymphs often undergo regressive molt (molt of a nymph to a pseudergate) in some termite species. In $H$. sjostedti, nymphs whose wing buds were mutilated were often found in relatively large and old colonies, and the regressive molt from nymphs to pseudergates was observed in the laboratory rearing experiments [31]. Such wing bud mutilation could be the selective pressure that led to the reduction of second-instar nymphs in $H$. sjostedti. In some species of Zootermopsis, reproductive soldiers or soldier neotenics were reported, and they are suggested to have evolved as a result of intra- or inter-colonial conflicts [32, 33]. In addition, it is also suggested that the caste differentiation pathways in this family (Archotermopsidae) are more plastic than those in other lineages. In this termite family, therefore, these preadaptive conditions, i.e., intragroup conflicts and developmental plasticity, might have facilitated the appearance of soldier neotenics or the heterochronic reduction of nymphal instars.

\section{Conclusion}

In this study, we obtained information about the unique alate differentiation of $H$. sjostedti from the perspectives of histology and gene expression. Our findings suggested that the developmental events for wing formation are compacted into a single nymphal instar in $H$. sjostedti, 
and as a result, a unique wing formation is seen to compensate for the spatial restriction inside small wing buds, leading to the completion of functional wings. It is suggested that there are behavioral or ecological factors, such as intracolony conflicts among the reproductives, underlying the reduction of nymphal instars. In the future, further studies including behavioral observations will be necessary to get insight into the evolutional process in the reduction of nymphal instars.

\section{Methods}

\section{Termites}

Colonies of $H$. sjostedti Holmgren and $Z$. nevadensis (Hagen) were collected on Yakushima Island, Kagoshima Prefecture, and at Kawanishi City, Hyogo Prefecture, Japan, respectively, in May 2016-2018. Z. nevadensis is known to be a species that was introduced to Japan [34]. Nest logs of both species were maintained in the laboratory as stock colonies at approximately $25^{\circ} \mathrm{C}$ under constant darkness, as described previously [11, 12, 35]. For their food, moistened pine lumber was occasionally supplied. In $H$. sjostedti, since alates are produced in June under natural conditions, many of the colonies sampled in May contain nymphs. In Z. nevadensis, the season for alate production is not so restricted, and the sampled colonies usually contain nymphs throughout most of the year.

\section{Histological observations}

Paraffin sections were made for histological observations on the developmental processes of wings in both species. The examined time points were first-instar nymph (N1) and early and late stage of second-instar nymph (N2E and N2L) in $Z$. nevadensis, and early, mid and late nymphal stage (NE, NM and NL) in H. sjostedti. Dissected thoracic parts of nymphs were fixed in FAA fixative (formaldehyde/acetic acid/ethanol $=6: 1: 16$ ) and preserved in $70 \%$ ethanol. Samples were dehydrated in increasing concentrations of ethanol, then transferred into xylene and finally embedded into paraffin. Serial section ( $8 \mu \mathrm{m}$ thick) in sagittal and transverse planes was processed routinely and stained with hematoxylin and eosin according to the methods previously reported [36]. Tissues on slides were observed using a BZ-9000 HS All-in-one fluorescence microscope (KEYENCE, Osaka, Japan).

\section{Scanning electron microscopy}

Scanning electron microscopy was carried out to compare the wing bud structures of nymphs and the process of wing expansion during the imaginal molt between $Z$. nevadensis and $H$. sjostedti. This process was classified into 3 time points, i.e., prior to imaginal molt (PIM), early stage of imaginal molt (EIM), and late stage of imaginal molt (LIM). Thoracic parts of individuals before and after the imaginal molt were fixed in FAA. After fixation, the thoracic parts were transferred into increasing concentrations of ethanol followed by $t$-butanol as described in a previous report [12]. In samples at early stages of the imaginal molt, nymphal cuticles were easily removed for observations on newly formed wings. After the transfer into $t$-butanol, the fixed samples were freeze-dried using a Freeze Dryer ES-2030 (Hitachi Global, Tokyo, Japan), and coated with gold ions with an Ion Sputter E-1010 (Hitachi Global, Tokyo, Japan). Samples were observed with a JSM-5510LV scanning electron microscope (JEOL Ltd., Tokyo, Japan).

\section{Identification of wing-patterning and hormone-related genes}

For the comparison of wing development between the two termite species, orthologs of the wing development genes and hormone-related genes were searched in the genome sequence database of $Z$. nevadensis (http:// termitegenome.org; [37]) and the transcriptome data of H. sjostedti (DDBJ Sequence Read Archive: DRA005483; Sugime et al., [38].). As a result, 5 genes (ap [apterous], $d p p$ [decapentaplegic], vg [vestigial], Scr [Sex-combs reduced], exd [extradenticle]) were identified as wing development genes and 9 genes (Met [Methoprene-tolerant], Kr-h1 [Krüppel homolog 1], BR-C [Broad-Complex], EcR [Ecdysone Receptor], USP [ultraspiracle], E93, E75, InR2 [Insulin Receptor 2], FOXO [Forkhead box O]) were identified as hormone-related genes. The protein sequences of those candidate genes in Drosophila melanogaster were used as queries for tBLASTn searches against the datasets of the two species. Based on the obtained sequences, primers for real-time qRT-PCR were designed using Primer Express software (ver. 3.0.0, Applied Biosystems, Foster City, CA, USA, Additional file 6: Table S4).

\section{RNA extraction and real-time qRT-PCR}

For the investigations on the expression patterns of genes involved in the wing formation, thoracic parts (prothorax, mesothorax and metathorax excluding guts and legs) were dissected from individuals, and immediately frozen in liquid nitrogen. The examined time points were pseudergate (PE), first-instar nymph (N1), early and late stage of second-instar nymph (N2E and N2L) and alate (A) in Z. nevadensis, and pseudergate (PE), early and late nymphal stage (NE and NL) and alate (A) in H. sjostedti. Frozen samples were individually preserved in $1.5 \mathrm{ml}$ polypropylene tubes at $-80{ }^{\circ} \mathrm{C}$ until RNA extraction. Total RNA was extracted using RNAiso Plus (Takara Bio, 
Shiga, Japan) according to the manufacturer's protocol. After the extraction, samples were treated with DNaseI (Thermo Fisher Scientific, Waltham, MA, USA).

For each sample, $900 \mathrm{ng}$ of total RNA was reversetranscribed with a High Capacity cDNA Reverse Transcription Kit according to the manufacturer's instructions (Applied Biosystems, Foster City, CA, USA). Quantifications of the relative amounts of target transcripts were then performed using a Fast SYBR Green Master Mix and the sequence detection system ABI PRISM 7500 (Applied Biosystems, Foster City, CA, USA). For identifying an appropriate endogenous control gene, the suitability of different candidate reference genes (18S rRNA, ribosomal protein L13a [RPL13a] and ribosomal protein 49 [RP49] in $Z$. nevadensis; $18 S$ rRNA, elongation factor 1 alpha [EF1a], and ribosomal protein 49 [RP49] in H. sjostedti) was evaluated using the software geNorm [39] and Normfinder [40]. As a result, RPL13a and RP49 were found to be the most appropriate reference genes for $Z$. nevadensis and $H$. sjostedti, respectively. Data acquisition and analyses were handled with ABI Prism 7500 software ver. 2.0.4 (Applied Biosystems, Foster City, CA, USA), with the relative standard curve method. For statistical analyses to detect significant differences of expression levels, respectively, in each species, Tukey's multiple comparisons test $(P<0.05)$ was performed after one-way ANOVA $(P<0.05)$, using $\mathrm{R}$ 3.5.2 (https://www.r-project.org). The number of samples was $n=5$ for all the samples of $Z$. nevadensis, and $n=3$ (PE, A) and $n=7$ (NE, NL) for H. sjostedti.

\section{Principal component analysis}

To examine the correspondence of nymphal stages between the two focal stages based on gene expression patterns, principal component analysis (PCA) was performed. In the analysis, hypothetical models of nymphal stages were made based on the stages of $H$. sjostedti, and were compared with the stages in $Z$. nevadensis. Firstly, the gene expression data that were standardized in the respective species to match the expression ranges among genes, were used for PCA on the correlation matrix to unify the respective gene expression patterns as principal component scores. Thereafter, the respective principal component scores were analyzed by the generalized linear model (GLM) with the Gaussian distribution with identity link. According to the Kaiser's criterion [41], 4 principal components were selected (Additional file 1: Fig. S1). Species and nymphal stages were used as the explanatory variables. The significance of the respective coefficients was examined by the Wald test. The best model was chosen in accordance with the Akaike's information criterion (AIC).

\section{Additional files}

Additional file 1. Fig. S1 The scree plot for the principal component analysis. Based on the the Kaiser's criterion (dotted line), 4 principal components were selected.

Additional file 2. Table S1. Cumulative proportions and principal component loadings in the principal component analysis targeting the gene expression levels in Zootermopsis nevadensis and Hodotermopsis sjostedti. Four principal components were selected in accordance with the Kaiser's rule. Bold letters indicate the value higher than 0.4.

Additional file 3. Fig. S2. Biplots of the principal component scores shown in Table $\mathrm{S} 1$. The principal components being $\mathrm{X}$ or $\mathrm{Y}$ axes were selected based on the results of the GLM analysis shown in Table S2. The red dotted circles indicate the plots of NL and A in Hodotermopsis sjostedti or N2L and A in Zootermopsis nevadensis. The gray dotted circles indicate the other stages.

Additional file 4. Table S2. Statistical results of the three models hypothesizing the growth stages in Zootermopsis nevadensis to match those in Hodotermopsis sjostedti. The asterisks indicate the statistical significances of the respective parameters tested by the Wald test $(n=45 ; P<0.05)$. The hypothetical growth stages in the best model independent from species differences are shown as bold. PE, workers; NE, early nymphal stage; $\mathrm{NL}$, late nymphal stage; $A$, alate.

Additional file 5. Table S3. Candidate genes focused in this study. Additional file 6. Table S4. Primer sequences for real time quantitative RT-PCR.

\section{Authors' contributions}

$\mathrm{RN}$ and TM designed the study outline. RN and KO performed the experiments and analyzed the obtained data. JS performed the statistical analyses. RN, $\mathrm{KO}$, JS, SK and TM wrote the manuscript. All authors read and approved the manuscript.

\section{Acknowledgements}

We would like to express our gratitude to Run Minoura, Hiroyuki Shimoji, Masaru K. Hojo and Yudai Masuoka for their assistance in field sampling.

\section{Competing interests}

The authors declare that they have no competing interests.

\section{Availability of data and materials}

Indicated in the text.

Consent for publication

Not applicable.

Ethics approval and consent to participate

Not applicable.

Funding

This work was supported by a Grant-in-Aid for Scientific Research A (No. 25251041) to TM and by Grant-in-Aid for JSPS Fellows to KO (No. 17J06879), from the Ministry of Education, Culture, Sports, Science and Technology of Japan.

\section{Publisher's Note}

Springer Nature remains neutral with regard to jurisdictional claims in published maps and institutional affiliations.

\section{Author details}

${ }^{1}$ Graduate School of Environmental Science, Hokkaido University, Sapporo, Hokkaido 060-0810, Japan. ${ }^{2}$ Misaki Marine Biological Station, School of Science, The University of Tokyo, Miura, Kanagawa 238-0225, Japan. ${ }^{3}$ Faculty of Environmental Earth Science, Hokkaido University, Sapporo, Hokkaido 060-0810, Japan 
Received: 2 February 2019 Accepted: 16 April 2019

Published online: 16 May 2019

\section{References}

1. Wilson EO. The insect societies. Cambridge: Belknap Press of Harvard University Press; 1971. p. 548.

2. Miura T. Developmental regulation of caste-specific characters in socialinsect polyphenism. Evol Dev. 2005;7:122-9.

3. Roisin Y. Diversity and evolution of caste patterns. In: Abe T, Bignell DE, Higashi M, editors. Termites: evolution, sociality, symbioses, ecology. Dordrecht: Kluwer; 2000. p. 95-119.

4. Noirot C. Pathways of caste development in the lower termites. In: Watson JAL, Okot-Kotber BM, Noirot C, editors. Caste differentiation in social insects. New York: Pergamon Press; 1985. p. 41-57.

5. Thompson GJ, Kitade O, Lo N, Crozier RH. Phylogenetic evidence for a single, ancestral origin of a 'true' worker caste in termites. J Evol Biol. 2000;13:869-81.

6. Korb J, Hartfelder K. Life history and development: a framework for understanding developmental plasticity in lower termites. Biol Rev. 2008:83:295-313.

7. Roisin Y, Korb J. Social organisation and the status of workers in termites. In: Bignell DE, Roisin Y, Lo N, editors. Biology of termites: a modern synthesis. Dordrecht: Springer; 2010. p. 133-64.

8. Legendre F, Whiting MF, Grandcolas P. Phylogenetic analyses of termite post-embryonic sequences illuminate caste and developmental pathway evolution. Evol Dev. 2013;15:146-57.

9. Noirot C. Formation of castes in the higher termites. In: Krishna K, Weesner FM, editors. Biology of termites, vol. 1. New York: Academic; 1969. p. 311-50.

10. Castle GB. The damp-wood termites of the western United States, genus Zootermopsis (formerly, Termopsis). In: Kofoid GA, editor. Termite and termite control. Berkeley: University of California Press; 1934. p. 273-310.

11. Miura T, Hirono Y, Machida M, Kitade O, Matsumoto T. Caste developmental system of the Japanese damp-wood termite Hodotermopsis japonica (Isoptera: Termopsidae). Ecol Res. 2000;15:83-92.

12. Miura T, Koshikawa S, Machida M, Matsumoto T. Comparative studies on alate wing formation in two related species of rotten-wood termites: Hodotermopsis sjostedti and Zootermopsis nevadensis (Isoptera, Termopsidae). Insectes Soc. 2004;51:247-52.

13. Weatherbee SD, Halder G, Kim J, Hudson A, Carroll S. Ultrabithorax regulates genes at several levels of the wing-patterning hierarchy to shape the development of the Drosophila haltere. Genes Dev. 1998;12:1474-82.

14. Abouheif E, Wray GA. Evolution of the gene network underlying wing polyphenism in ants. Science. 2002:2975579:249-52.

15. Brisson JA, Ishikawa A, Miura T. Wing development genes of the pea aphid and differential gene expression between winged and unwinged morphs. Insect Mol Biol. 2010;19:63-73.

16. King-Jones K, Thummel CS. Nuclear receptors-a perspective from Drosophila. Nat Rev Genet. 2005;6:311.

17. Lozano J, Belles X. Conserved repressive function of Krüppel homolog 1 on insect metamorphosis in hemimetabolous and holometabolous species. Sci Rep. 2011;1:163.

18. Charles JP, Iwema T, Epa VC, Takaki K, Rynes J, Jindra M. Ligand-binding properties of a juvenile hormone receptor; Methoprene-tolerant. Proc Natl Acad Sci. 2011:108:21128-33.

19. Belles X, Santos CG. The MEKRE93 (Methoprene tolerant-Krüppel homolog 1-E93) pathway in the regulation of insect metamorphosis, and the homology of the pupal stage. Insect Biochem Mol Biol. 2014;52:60-8.

20. Kayukawa T, Jouraku A, Ito Y, Shinoda T. Molecular mechanism underlying juvenile hormone-mediated repression of precocious larval-adult metamorphosis. Proc Natl Acad Sci. 2017;114:1057-62.

21. Goodman WG, Granger NA. In: Gilbert LI, latrou K, Gill SS, editors. Comprehensive molecular insect science, vol. 3. Oxford: Elsevier; 2005. p. 319-408.
22. Nijhout HF, Wheeler DE. Juvenile hormone and the physiological basis of insect polymorphisms. Q Rev Biol. 1982;57:109-34.

23. Cornette R, Gotoh H, Koshikawa S, Miura T. Juvenile hormone titers and caste differentiation in the damp-wood termite Hodotermopsis sjostedti (Isoptera, Termopsidae). J Insect Physiol. 2008;54:922-30.

24. Miura T, Scharf ME. Molecular basis underlying caste differentiation in termites. In: Bignell DE, Roisin Y, Lo N, editors. Biology of termites: a modern synthesis. Dordrecht: Springer; 2011. p. 211-53.

25. Konopova B, Smykal V, Jindra M. Common and distinct roles of juvenile hormone signaling genes in metamorphosis of holometabolous and hemimetabolous insects. PLoS ONE. 2011;6:e28728.

26. Smykal V, Daimon T, Kayukawa T, Takaki K, Shinoda T, Jindra M. Importance of juvenile hormone signaling arises with competence of insect larvae to metamorphose. Dev Biol. 2014;390:221-30.

27. Roisin Y. Morphology, development and evolutionary significance of the working stages in the caste system of Prorhinotermes (Insecta, Isoptera). Zoomorphology. 1988;107:339-47.

28. Parmentier D, Roisin Y. Caste morphology and development in Termitogeton nr. planus (Insecta, Isoptera, Rhinotermitidae). J Morphol. 2003:255:69-79.

29. Roisin Y, Pasteels JM. Replacement of reproductives in Nasutitermes princeps (Desneux) (Isoptera: Termitidae). Behav Ecol Sociobiol. 1986;18:437-42.

30. Roisin Y. Intragroup conflicts and the evolution of sterile castes in termites. Am Nat. 1994;143:751-63.

31. Koshikawa S, Matsumoto T, Miura T. Regressive molt in the Japanese damp-wood termite Hodotermopsis japonica (Isoptera: Termopsidae). Sociobiology. 2001;38:495-500.

32. Myles TG. Reproductive soldiers in the Termopsidae (Isoptera). Pan-Pac Entomol. 1986;62:293-9.

33. Thorne BL, Breisch NL, Muscedere ML. Evolution of eusociality and the soldier caste in termites: influence of intraspecific competition and accelerated inheritance. Proc Natl Acad Sci. 2003;100:12808-13.

34. Morimoto K. On the damp-wood termite genus Zootermopsis introduced to Japan. Shiroari. 2000;122:3-8 (in Japanese).

35. Miura T, Koshikawa S, Matsumoto T. Winged presoldiers induced by a juvenile hormone analog in Zootermopsis nevadensis: implications for plasticity and evolution of caste differentiation in termites. J Morphol. 2003;257:22-32.

36. Cornette R, Matsumoto T, Miura T. Histological analysis of fat body development and molting events during soldier differentiation in the dampwood termite, Hodotermopsis sjostedti (Isoptera, Termopsidae). Zool Sci. 2007:24:1066-74.

37. Terrapon N, Li C, Robertson HM, Ji L, Meng X, Booth W, Gowin J. Molecular traces of alternative social organization in a termite genome. Nat Commun. 2014:5:3636

38. Sugime Y, Oguchi K, Gotoh H, Hayashi Y, Matsunami M, Shigenobu S, Koshikawa S, Miura T. Termite soldier mandibles are elongated by dachshund under hormonal and Hox gene controls. Development 2019;146:dev171942

39. Vandesompele J, De Preter K, Pattyn F, Poppe B, Van Roy N, De Paepe A, Speleman F. Accurate normalization of real-time quantitative RT-PCR data by geometric averaging of multiple internal control genes. Genome Biol. 2002;3:research0034-1.

40. Andersen $\mathrm{CL}$, Jensen JL, Ørntoft TF. Normalization of real-time quantitative reverse transcription-PCR data: a model-based variance estimation approach to identify genes suited for normalization, applied to bladder and colon cancer data sets. Cancer Res. 2004:64:5245-50.

41. Kaiser HF. The application of electronic computers to factor analysis. Educ Psychol Meas. 1960;20:141-51. 